\title{
Freeze Drying Method with Gaseous Nitrogen to Preserve Fine Ultrastructure of Biological Organizations for Scanning Electron Microscopy, Helium Ion Beam Microscopy and Fluorescence Microscopy
}

\author{
Kunihiro Uryu ${ }^{1}$, Nadine Soplop ${ }^{1}$, Devrim Acehan ${ }^{1}$, Benjamin Y. Winer ${ }^{2}$, Vincent A. Fischetti ${ }^{2}$, Timothy \\ Sheahan $^{3}$, Charles M. Rice ${ }^{3}$, Mayla Hsu ${ }^{4}$, Melissa Robbiani ${ }^{4}$, Gaetano Santulli ${ }^{5}$, Hana Totary-Jain ${ }^{5,6}$, Bernhard \\ Goetze $^{7}$, Maria-Teresa Catanese ${ }^{3,8}$
}

${ }^{1}$ Electron Microscopy Resource Center, ${ }^{2}$ Laboratory of Bacterial Pathogenesis and Immunology,

${ }^{3}$ Laboratory of Virology and Infectious Disease, The Rockefeller University, New York, New York, USA; ${ }^{4}$ Center for Biomedical Research, Population Council, New York, New York, USA, ${ }^{5}$ Department of Physiology and Cellular Biophysics, The Clyde and Helen Wu Center for Molecular Cardiology, Columbia University Medical Center, New York, New York, USA., ${ }^{6}$ Department of Molecular Pharmacology \& Physiology, University of South Florida Morsani College of Medicine, Tampa, Florida, USA., ${ }^{7}$ Ion Microscopy Innovation Center, Zeiss Microscopy LLC, Peabody, Massachusetts, USA., ${ }^{8}$ Department of Infectious Diseases, King's College London School of Medicine, Guy's Hospital, Great Maze Pond, London SE1 9RT, UK

Precise demonstration of the surface architecture of biological organisms is critical to elucidate functional events taking place on the cell membranes. Cell membranes provide refined and orchestrated molecular arrangements enabling biological organism to conduct their sophisticated physiological activities ${ }^{1}$. Scanning Electron Microscopy (SEM) has great potential to visualize detailed structures associated with dynamic events on the surface of materials. Dry hard materials, such as graphene or gold, are readily applicable to a high vacuum chamber with an electron beam and yield highly detailed information at sub-nano scale. In sharp contrast, biological organisms, with lipid-rich membranes encompass cytoplasm containing $>80 \%$ of water, are extremely challenging objects to apply for SEM. To apply such biological organisms, ethanol dehydration was developed based on the assumption that ethanol was a mild organic solvent that caused less damage than most organic solvents, while concerns over the loss of lipids prompted a search for alternative reagents or methodology $y^{2,3}$. The application of osmium tetroxide to fix unsaturated lipids ${ }^{4}$, variable derivatives of organic solvents, or lowering ethanol temperature were introduced to minimize the loss 5 . Lipid loss during EM processing was examined and believed to be minor, nevertheless these assessments were limited to biochemical means and the question regarding structural integrity remained unanswered. Despite such uncertainty, the existing ethanol dehydration step for SEM sample preparation is widely used.

Given these considerations, we carried out a series of tests for SEM methodological development, aiming to achieve the following goals; 1) retaining the integrity of lipid-rich membranes, 2) attaining high resolving power up to nm scale, 3) retaining activity of fluorescent proteins, and 4) enabling applicability to a broad range of biological samples. We came up with a three-step-procedure: 1) plunge freezing for instantaneous immobilization; 2) freeze drying in a cold nitrogen gas $\left(\mathrm{GN}_{2}\right)$; and 3 ) critical point drying (CPD). Here we describe a new sample preparation procedure, coupled with a freeze dry method using gaseous nitrogen $\left(\mathrm{FDGN}_{2}\right.$ method) resulting in unprecedented structural detail using high vacuum SEM.

Extended assessments revealed that the $\mathrm{FDGN}_{2}$ method was adoptable to Helium Ion beam Microscopy (HIM) ${ }^{6}$. Recent development of gas-filed-ionization source, reduced aberrations and charge compensation electron flood gun made HIM potentially a super-resolution scanning microscope of native topographic architecture without conductive coating. However, successful biological applications have been limited and it awaits further methodological supports. Further assessment revealed that the 
FDGN $_{2}$ method retains the activity of fluorescent proteins as well as reasonable ultrastructure in thin sections for Transmission Electron Microscopy (TEM).

The new information presented with the $\mathrm{FDGN}_{2}$ method using multimodal imaging systems exemplified a reliable complementary structural assessment for highly sophisticated biological organisms. [7]

\section{References:}

[1] GW Hart, RJ Copeland, Cell 143, (2010), p. 672.

[2] CM Krueger, EJ Neufeld, JE Saffitz, J Histochem Cytochem 33 (1985), p. 799.

[3] A Ongun, WW Thomson, JB Mudd, J Lipid Res 9 (1968), p. 416.

[4] DR Saunders, J Wilson, CE Rubin, J. Cell Biol. 37 (1968), p 183.

[5] RD Dallam, J Histochem Cytochem 5 (1987), p. 178.

[6] MS Joens, C Huynh, JK Kasuboski et al. Sci. Rep. 3 (2013), p. 3514.

[7] Acknowledgements: The current study was supported by The Rockefeller University, the American Heart Association Scientist Developmental Grant 15SDG25300007 (Santulli), the United States Agency for International Development Cooperative Agreement GPO-A-00-04-00019-00 (Robbiani), the NIH grant AI098645 (Robbiani), the TNPRC NIH base grant P51 OD011104-52 (Robbiani), NIH
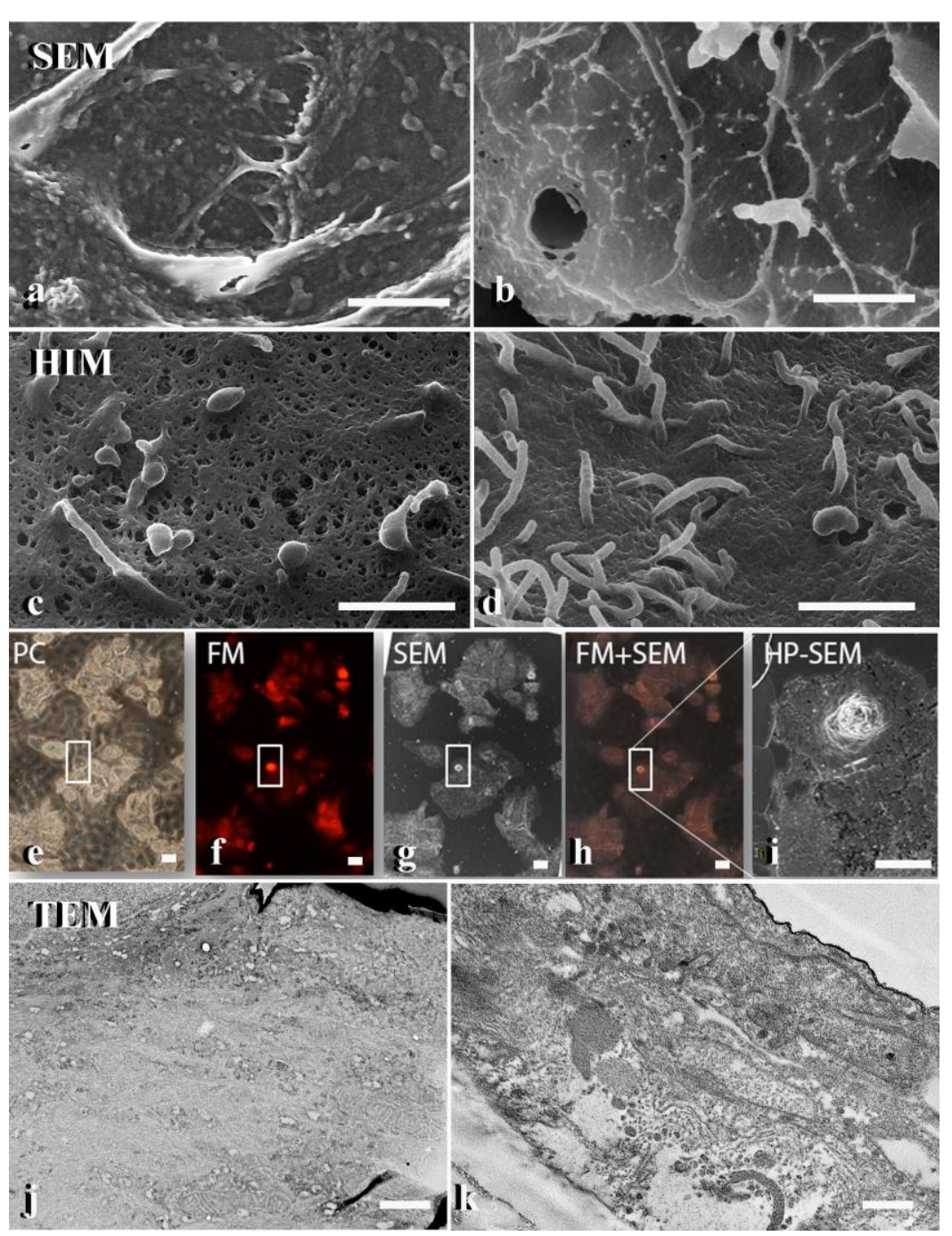

R01 AI072613 (Rice), NIH R01 CA057973 (Rice), NHLBI/NIH R00HL109133 (Totary-Jain) and ContraFect Corporation (Fischetti).

Figure: Comparison of SEM views $(a, b)$ of human hepatoma cells prepared by two methods, ethanol (a) or $\mathrm{FDGN}_{2}$ based (b) method, indicated overt difference in detail presentation. The identical comparison was made (ethanol (c) or $\mathrm{FDGN}_{2}$ based method (d)) for HIM examination (c,d). Post-FDGN 2 dehydration the cells were also examined by phase contrast microscopy (PC, e), fluorescent microscopy (FM, f), and SEM at low power (g) or high power (i). FM and SEM images were superimposed (h). After SEM examinations the cells and rat carotid artery prepared by $\mathrm{FDGN}_{2}$ methods were processed for resin embedding, ultrathin sectioning and examined by TEM. Reasonable ultrastructure was presented. Dark outline represent conductive coating used for SEM. Scale bars represent 50nm, except for e-i $(10 \mu \mathrm{m})$. 